\title{
Magnetic Shields for Underground Power Lines
}

\author{
J.R. Riba Ruiz ${ }^{1}$, X. Alabern Morera ${ }^{2}$ \\ ${ }^{1}$ Department d'Enginyeria Elèctrica, UPC \\ EUETII-"L'Escola d'Adoberia" \\ Plaça del Rei 15, 08700 Igualada (Spain) \\ phone:+34 938 035300, fax:+34 938 031589, e-mail: jordi@euetii.upc.es \\ ${ }^{2}$ Department d'Enginyeria Elèctrica, UPC \\ C./ Colom 11, 08222 Terrassa (Spain) \\ phone: +34 937 398035, alabern@ee.upc.es
}

\begin{abstract}
This paper describes a simple method for shielding the magnetic fields generated by underground power lines. It consists on placing a cylindrical shell made of a ferromagnetic material. The principle for reducing the magnetic field outside the shield is that the ferromagnetic material absorbs the magnetic field generated by the buried power line and only a very small portion of the magnetic field survives outside the shield. As the shield is made of a high-conductivity ferromagnetic material, eddy currents are induced inside it and they generate a magnetic field opposed to the magnetic field generated by the power line. It results that the total magnetic field $H$ inside the shield is reduced.

It is well known that the relative magnetic permeability of ferromagnetic materials decreases sharply when magnetic fields intensities $H$ are very weak (below $0,1 \mathrm{~A} / \mathrm{m}$ ) and when they are very strong (due to saturation phenomena). In these situations the shielding efficiency of these materials is dramatically reduced.

The selection of an adequate material to act as a magnetic field is decisive. Such a material must have a high magnetic permeability when dealing with very weak magnetic fields and a high electric conductivity in order to maximize the absorption losses. For these reasons the selected material has been the purified iron (at least 99'95\% pureness).
\end{abstract}

\section{Key words}

Magnetic field, shielding, ferromagnetic materials, absorption losses, underground power lines.

\section{Introduction}

Underground power lines create magnetic fields in their neighbourhood. These fields can produce electromagnetic interferences in electric and electronic equipments placed near the power lines. While some medical studies conclude that electric and magnetic fields generated by power lines are not harmful for human beings, other studies conclude that they can cause some diseases in human beings. For these reasons we think that in the future the magnetic shielding of power underground lines will be a reality.

The magnetic fields generated by underground power lines have intensity values of some $\mu \mathrm{T}$ in the vertical of the line ( $1 \mathrm{~m}$ above the floor surface). The magnetic field decreases sharply with the distance to the line.

Due to the low intensities of the magnetic fields generated by the underground power lines, it is very important to select a suitable material for the shield. In order to create an efficient magnetic shield, the materials suitable for building the shield should have a very high magnetic permeability value.

When dealing with weak magnetic field intensities the selection of the material of the shield is crucial because many of the usual ferromagnetic materials have relative magnetic permeability which value is very close to the unit. This means that the former materials are not suitable to mitigate the magnetic fields generated by underground power lines.

The materials that are suitable for this purpose must have a very high electric conductivity and a high initial magnetic permeability.

We have found that purified iron ${ }^{[1]}$ combines a very high electric conductivity with high permeability values for low magnetic fields.

In this work we realize a comparative study of the magnetic field mitigation due to different geometries of the magnetic shields. Calculations have been carried out by applying the finite elements method.

\section{The magnetic shields geometry}

In this work we study an underground power line of 25 $\mathrm{kV}$. This line has three active conductors that carry a system of 100 A balanced currents. The longitudinal axes of the three parallel conductors are separated $10 \mathrm{~cm}$. 
The conductors are placed in the same horizontal plane, one meter below the floor surface.

Figure 1 shows the ditch and the three underground conductors.

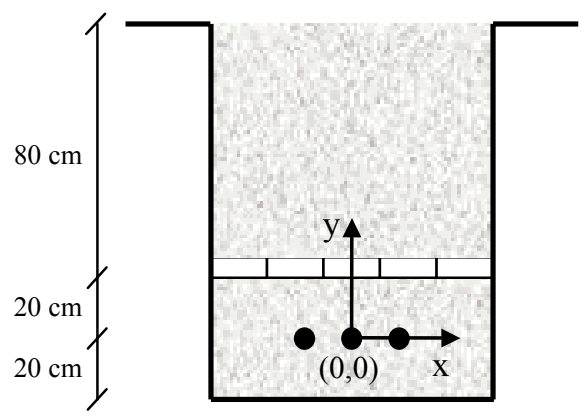

Figure 1. Ditch and position of the underground conductors.

The proposed shield of the magnetic field, has a thickness of $2 \mathrm{~mm}$ and their geometric dimensions are shown in the next figure.

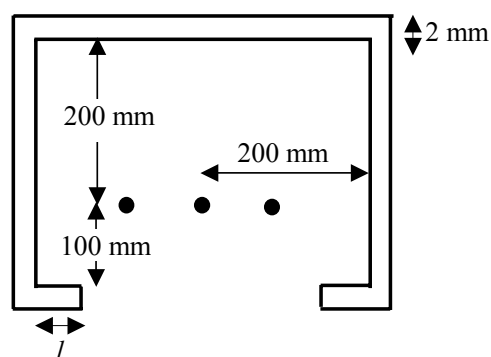

Figure 2. Magnetic field shield.

In this work, four different geometries of magnetic shields have been compared. These geometries are shown in figure 2 and have arms-length $l=0,50,100,150$ and $200 \mathrm{~mm}$.

The material selected to create the magnetic shield has been purified iron (99'95\% pureness). This is due to the fact that purified iron has a high magnetic permeability when dealing with weak magnetic field intensities (in the calculations we have supposed $\mu_{\mathrm{r}}=500$ ) and also has a high electric conductivity and reduced hysteresis losses.

TABLE I. Electric and magnetic properties for purified iron ${ }^{[1]}$.

\begin{tabular}{cc}
\hline Material & Purified Iron \\
Electric conductivity & $\sigma=10^{7} \mathrm{~S} / \mathrm{m}$ \\
Relative permeability $(\mathrm{B}=2 \mathrm{mT})$ & $\mu_{\mathrm{r}}=5000$ \\
Maximum relative permeability & $\mu_{\mathrm{r}, \text { max. }}=180000$ \\
Skin depth & $\delta=1,01 \mathrm{~mm}$ \\
Hysteresis losses* & $30 \mathrm{~J} / \mathrm{m}^{3}$ \\
Density & $7880 \mathrm{~kg} / \mathrm{m}^{3}$ \\
\hline
\end{tabular}

* In magnetic saturation conditions

When dealing with a fixed frequency, the magnetic field reduction factor is very dependent on the skin depth $\delta$. The skin depth is one of the most important factors to bear in mind when designing a magnetic field shield.
The skin depth can be calculated from the following expression:

$$
\delta=1 / \sqrt{\pi f \mu \sigma}
$$

being $f$ the electric network frequency expressed in $\mathrm{Hz}, \mu$ the absolute permeability of the shield material expressed in $\mathrm{H} / \mathrm{m}$, and $\sigma$ its electric conductivity expressed in $(\Omega . \mathrm{m})^{-1}$.

\section{The magnetic shields geometry}

The mathematical simulations have been carried out by means of the finite elements free software femm 3.2 ${ }^{[2]}$. A total approximate number of 35000 nodes and 75000 elements have been created in each simulation. A circular section with a diameter of $15 \mathrm{~m}$ centered in the geometric center of the conductors has been simulated, as shown in figure 3 .

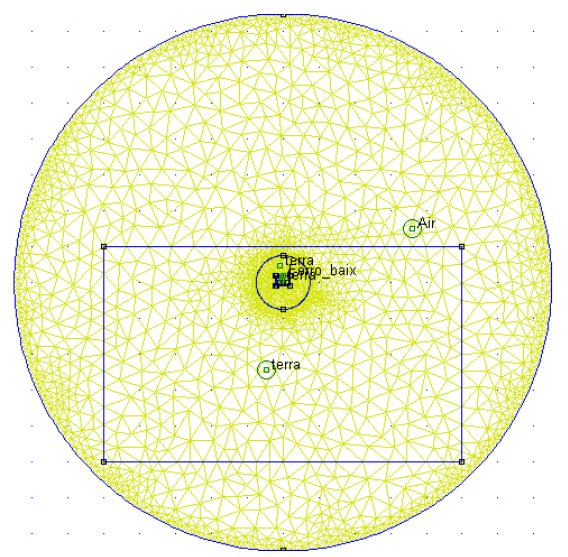

Figure 3. Geometry and mesh of the simulations.

The magnetic field reducing factor $\left(R F_{\%}\right)$ is defined as:

$$
R F_{\%}=100 \cdot \frac{B_{o}-B}{B_{o}}
$$

where $B_{o}$ is the magnetic field intensity in any point of the space when there is not present the magnetic shield, while $B$ is the magnetic field intensity in the same point when the shield is present. If due to the shied, the magnetic field is reduced, the values of the magnetic field reducing factor will be positive, but if there was an increment of the magnetic field, their values would be negative.

Figure 4 shows the areas where the magnetic fields and the reducing factor values have been computed.

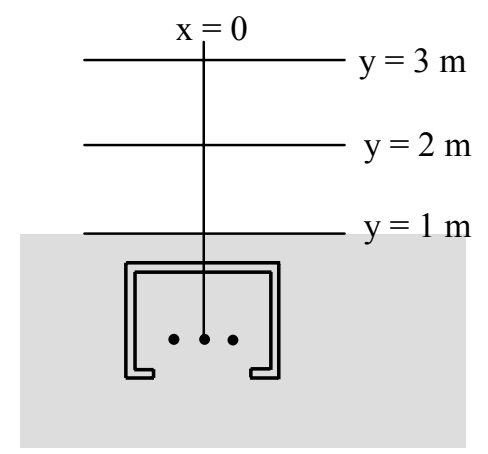

Figure 4. Geometry and reference axes. 


\section{Results}

In all the simulations a constant value $\mu_{r}=500$ of the magnetic permeability of the pure iron shield has been supposed. All the simulations have been carried out by supposing a shield thickness of $2 \mathrm{~mm}$. By incrementing the thickness of the shields, a better magnetic field reducing factor will be obtained.

We have realized a total of 5 simulations. Each simulation has been carried out by considering a different length of the arms of the shield. The lengths we have dealt with are $0 \mathrm{~mm}, 50 \mathrm{~mm}, 100 \mathrm{~mm}, 150 \mathrm{~mm}$ and 200 $\mathrm{mm}$.

Figure 5 shows a 3D plot of the magnetic field distribution generated by the underground power line when the magnetic shield is not present.

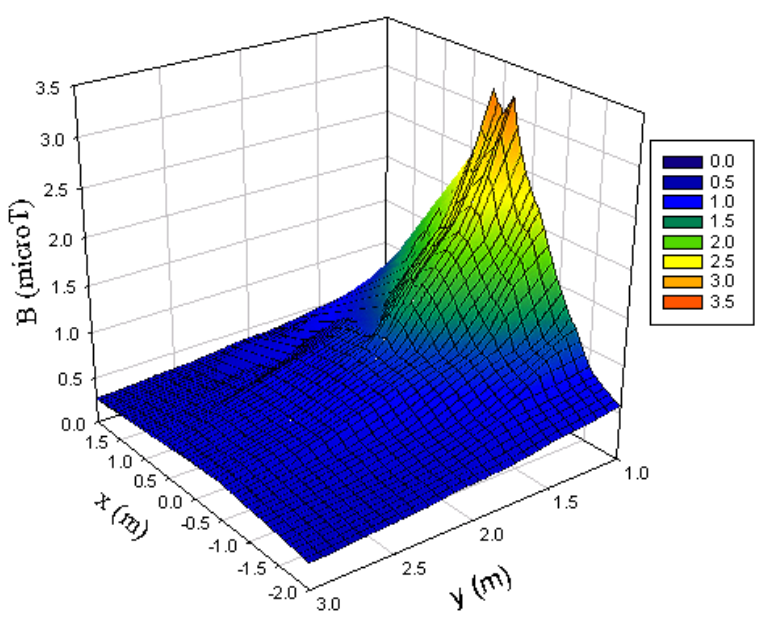

Figure 5. Magnetic field distribution without the shield.

Figure 6 shows a 3D plot of the magnetic field distribution generated by the underground power line when a $2 \mathrm{~mm}$-thick shield with arms of $200 \mathrm{~mm}$-length is present.

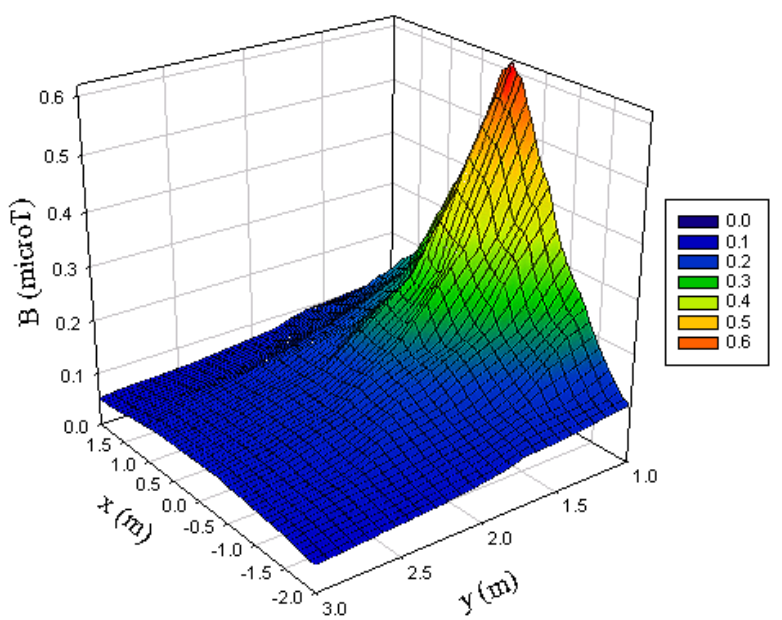

Figure 6. Magnetic field distribution with a shield of 200 mm-length arms.

From figures 5 and 6 it can be deduced than in the areas where the magnetic field is more intense, when dealing with a $2 \mathrm{~mm}$-thick shield with arms of $200 \mathrm{~mm}$, the magnetic field has been reduced by a factor of 5 .
Figure 7 shows the magnetic field reducing factor distribution in the $X Y$ plane. The two overlap figures suppose a thickness of $2 \mathrm{~mm}$ and arms-length of $0 \mathrm{~mm}$ and $200 \mathrm{~mm}$ (shield closed).

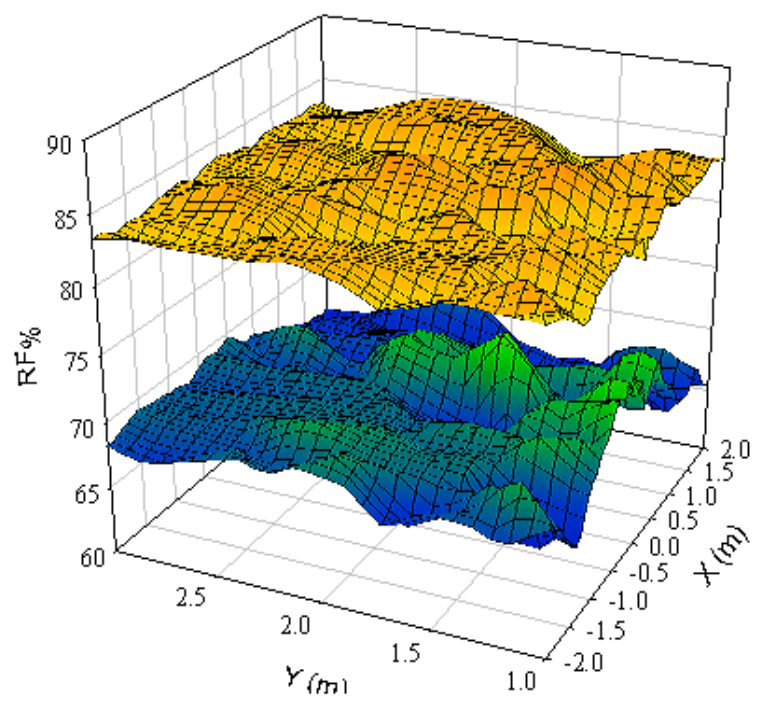

Figure 7. Comparison of the magnetic field reducing factor for two different shields geometries (up: $l=0 \mathrm{~mm}$, bottom: $l=200 \mathrm{~mm}$ ).

Tables II shows the average magnetic field reducing factors, for the vertical plane placed $1 \mathrm{~m}$ above the floor surface.

TABLE II. Average values of the magnetic field reducing factor, computed $1 \mathrm{~m}$ above floor surface.

\begin{tabular}{ccc}
\hline Arms-length & Average $\mathrm{RF}_{\%}$ & Average B $(\mu \mathrm{T})$ \\
\hline No shield & 0 & 0,747 \\
$0 \mathrm{~mm}$ & 68,6 & 0,231 \\
$50 \mathrm{~mm}$ & 75,4 & 0,181 \\
$100 \mathrm{~mm}$ & 79,7 & 0,149 \\
$150 \mathrm{~mm}$ & 81,5 & 0,136 \\
$200 \mathrm{~mm}$ & 84,0 & 0,120 \\
\hline
\end{tabular}

Table III shows the average magnetic field reducing factors computed in the vertical of $x=0 \mathrm{~m}$, from the floor surface $(y=1 \mathrm{~m})$ until 2 meters above the floor surface $(y$ $=3 \mathrm{~m}$ ).

TABLE III. Average values of the magnetic field reducing factor computed in the vertical of the central wire.

\begin{tabular}{ccc}
\hline Arms-length & Average $\mathrm{RF}_{\%}$ & Average B $(\mu \mathrm{T})$ \\
\hline No shield & 0 & 1,236 \\
$0 \mathrm{~mm}$ & 69,8 & 0,367 \\
$50 \mathrm{~mm}$ & 76,9 & 0,286 \\
$100 \mathrm{~mm}$ & 81,1 & 0,239 \\
$150 \mathrm{~mm}$ & 82,5 & 0,222 \\
$200 \mathrm{~mm}$ & 83,4 & 0,210 \\
\hline
\end{tabular}

From the results shown in table III one can deduce that if the objective is to obtain a magnetic field reducing factor greater than $80 \%$, it is necessary that the arms-length be at least $100 \mathrm{~mm}$. 


\section{Power losses}

The magnetic shield is made of a material with good electric conductivity. It means that in the shield will be generated eddy currents that will cause a heating of the shield.

As the shield is made of ferromagnetic material, extra heating losses due to the hysteresis loops will be produced.

The two phenomena are due to the oscillating magnetic field generated by the power line and they cause a general heating of the shield.

In this section the power losses due to this heating are computed. It will be shown that the value of these losses is very reduced.

The values of the resistive losses (due to eddy currents) are directly calculated for the finite elements program femm 3.2.

The hysteresis losses will be computed by an approximated method. We will suppose that the ferromagnetic material is not saturated. This hypothesis is valid because the magnetic field generated by the power line is very weak, less than $1 \mathrm{mT}$.

It is possible to estimate the hysteresis losses by the following expressions:

$$
P_{\text {Hist. }}\left(W / m^{3}\right)=f \cdot \int H \cdot d B \approx 2 \cdot f \cdot \bar{B} \cdot \bar{H}
$$

being $\bar{B}$ the average values of the magnetic field inside the shield.

Since $B=\mu_{o} \cdot \mu_{r} \cdot H$, the former expression can be transformed in:

$$
\begin{gathered}
P_{\text {Hist. }}\left(W / m^{3}\right)=\frac{2 \cdot f \cdot \bar{B}^{2}}{\mu_{o} \cdot \mu_{r}} \\
p_{\text {Hist. }}(W / m) \approx \frac{2 \cdot f \cdot \bar{B}^{2}}{\mu_{o} \cdot \mu_{r}} \cdot S=1,59 \cdot 10^{5} \cdot \bar{B}^{2} \cdot S
\end{gathered}
$$

where $S$ is the cross-section of the shield expressed in $\mathrm{m}^{2}$.

TABLE I. Losses in the studied shields.

\begin{tabular}{cccc}
\hline $\begin{array}{c}\text { Arms } \\
\text { length }\end{array}$ & $\begin{array}{c}\text { Resistive } \\
\text { losses } \\
\left(10^{-1} \mathrm{~W} / \mathrm{m}\right)\end{array}$ & $\begin{array}{c}\text { Hysteresis } \\
\text { losses } \\
\left(10^{-2} \mathrm{~W} / \mathrm{m}\right)\end{array}$ & $\begin{array}{c}\text { Total } \\
\text { losses } \\
\left(10^{-1} \mathrm{~W} / \mathrm{m}\right)\end{array}$ \\
\hline $0 \mathrm{~mm}$ & 0,65 & 0,59 & 0,71 \\
$50 \mathrm{~mm}$ & 0,79 & 0,76 & 0,87 \\
$100 \mathrm{~mm}$ & 1,01 & 0,94 & 1,10 \\
$150 \mathrm{~mm}$ & 1,22 & 1,09 & 1,33 \\
$200 \mathrm{~mm}$ & 1,32 & 1,10 & 1,43 \\
\hline
\end{tabular}

\section{Conclusions}

Once the study has been realized, the following conclusions can be deduced:

- In the present work it has been shown that it is possible to reduce the magnetic fields values near the underground power lines more than a $80 \%$ by using a shield of appropriate ferromagnetic material.
- The simulations have been carried out by supposing a shield thickness of only $2 \mathrm{~mm}$. By using thicker shields it is reasonable to think that the magnetic field reduction factor will increase.

- From the obtained results it is possible to deduce that the effectiveness of the shield is increased with the length of the arms.

- The computed values of the total power losses (hysteresis and eddy currents) are fewer than $15 \mathrm{~W}$ each $100 \mathrm{~m}$ of underground power line length.

\section{References}

[1] Handbook of Chemistry and Physics, 70th. Edition. CRC Press, pp. E-128, 1989-90.

[2] David Meeker. Finite Element Method Magnetics (FEMM 3.2). User's Manual. It can be find in http://femm.foster-miller.net/index.html. October 25, 2001.

[3] Fahy S., Kittel C. y Louie S. "Electromagnetic screening by metals". Am. J. Phys. Vol 11, pp. 989 (1988).

[4] P. Rochon and N. Gauthier "Strong shielding due to an electromagnetically thin metal sheet". Am. J. Phys. Vol. 58, pp. 276 (1990).

[5] J.R. Riba, X. Alabern. "Reducción del campo magnético creado por líneas enterradas de alta tensión utilizando materiales no ferromagnéticos" in Proc. 8CLEE 2003, Vol. 1, pp. 1.149-1.154.

[6] Jordi-Roger Riba, Xavier Alabern. "Estudio de los campos magnéticos y eléctricos generados por una línea aérea de $220 \mathrm{kV}$ ". 7as. Jornadas "La Ingeniería Eléctrica y Ambiental en el siglo XXI", INGENA 2001. Proceedings, P071, pg. 219-223. Universidad de Cantabria, Santander, 2001.

[7] Jordi-Roger Riba, Xavier Alabern. "Campo magnético generado por las líneas de alta tensión. Efecto del grado de desequilibrio del sistema de corrientes trifásicas". 7as. Jornadas Hispano-Lusas de Ingeniería Eléctrica. Proceedings, Vol. II, pg. 4348. Universidad Carlos III, Leganés (Madrid), 2001.

[8] Gao Yougang, $\mathrm{Yu}$ Lifang. Determination of Dangerous Region of the Electromagnetic Pollution Caused by the Electric Fields around Power Lines. International Conference on Communication Technology (ICCT 98), pgs. S26-01-1 a S26-01-4, 22-24, October 1998, Beijing (China).

[9] P.S. Wong, M.A. Janoska, C. Light, R.W. McCourt. "Long Term Magnetic Field Monitoring Near Power Lines”. IEEE Transactions on Power Delivery, 12 (2), 922-927, 1997.

[10] W.T. Kaune, L.E. Zafanella. "Analysis of Magnetic Fields Produced Far From Electric Power Lines". IEEE Transactions on Power Delivery, 7 (4), 20822091, 1992.

[11] IARC. IARC finds limited evidence that residential magnetic fields increase risk of childhood leukaemia. www.iarc.fr, cie@iarc.fr, june 2001. 